\title{
Knee Injury and Osteoarthritis Outcome Score
}

National Cancer Institute

\section{Source}

National Cancer Institute. Knee Injury and Osteoarthritis Outcome Score. NCI Thesaurus. Code C153299.

A self-reported instrument to assess a patient's opinion about their knee and associated problems. It consists of 5 subscales: Pain, Function in Daily Living, Function in Sport and Recreation, Other Symptoms, and Knee Related Quality of Life. 